\title{
DONNÉES PRÉLIMINAIRES SUR L'ÉCOLOGIE DE XENOPSYLLA CUNICULARIS SMIT, 1957 (SIPHONAPTERA, PULICIDAE) PARASITE DU LAPIN DE GARENNE ${ }^{1}$
}

\author{
H. LAUNAY
}

RÉSUMÉ. Une population de Xenopsylla cunicularis parasite du lapin de garenne Oryctolagus cuniculus a pu être suivie mensuellement pendant l'année 1977 dans le Sud-Ouest de la France. Les émergences massives d'adultes commencent au début du printemps et atteignent un maximum au début de l'été. La majeure partie des individus se trouvent alors sur le sol des terriers. La reproduction se termine au début de l'automne, saison qui est marquée par une mortalité très forte. Les survivants passent l'hiver préférentiellement sur l'hôte. Le cycle annuel de $X$. cunicularis est comparé à celui de Spilopsyllus cuniculi et son intérêt épidémiologique est souligné.

\section{On the phenology of the flea Xenopsylla cunicularis Smit, 1957 (Siphonaptera, Pulicidae) parasite of the European rabbit.}

SUMMARY. A population of Xenopsylla cunicularis parasitic on the European rabbit Oryctolagus cuniculus was studied monthly during the year 1977 in the South-West of France. The largescale emergence of adults take place at the beginning of summer. A major part of them is then found in the soil of the burrows. Reproduction lease at the beginning of autumn which is marked by a great mortality. Some fleas persiste in winter, and they are found on the hosts. The annual cycle of $X$. cunicularis is compared with that of Spilopsyllus cuniculi and the epidemiological interest is laid strees on.

\section{Introduction}

Xenopsylla cunicularis est très proche morphologiquement de $X$. ramesis (Rothschild, 1904), puce de Gerbillinae, avec laquelle elle a été confondue jusqu'à ce que Smit (1957) en fasse la diagnose. Mutant écologique, elle s'est adaptée à un lagomorphe : Oryctolagus cuniculus. En cela elle se distingue nettement des autres

Institut de Parasitologie de l'Ouest, Faculté de Médecine (Parasitologie : Entomologie médicale), F 35000 Rennes.

I Travail effectué grâce à l'aide financière de l'A.N.C.L.A.T.R.A. et à une subvention de l'Office national de la Chasse.

Accepté le 7 juillet r98r. 
Xenopsylla du groupe "conformis » auquel elle appartient et qui sont toutes parasites de rongeurs. Il paraît donc intéressant de voir si elle présente des modalités originales d'adaptation à son hôte dans son écologie et sa biologie, ou si au contraire elle a gardé les caractères généraux du groupe. Dans cette optique il est possible de la comparer :

- d'une part aux Xenopsylla du groupe "conformis " bien étudiées car elles jouent un grand rôle dans l'épidémiologie de la peste selvatique.

- d'autre part avec une autre puce spécifique du lapin de garenne, Spilopsyllus cuniculi adaptée d'une façon très particulière à son hôte, et bien étudiée elle aussi car intervenant dans l'épidémiologie de la myxomatose.

\section{I - Généralités}

\section{A - Répartition de Xenopsylla cunicularis}

X. cunicularis est connue du Maroc au sud de la France ( $f g$. . 1).

Au Maroc, sept stations, voisines, sont actuellement recensées : région de Rabat (Hopkins et Rothschild, 1953, sub. nom. ramesis, Smit 1957 emend.), forêt de Nefifikh (Blanc et Bruneau, 1956 et Grenier, 1957, sub. nom. ramesis, Smit 1957 emend.), forêt du Cherrat (Blanc et coll., 1962), région de Bouknadel (Rousselle, 1968, sub.

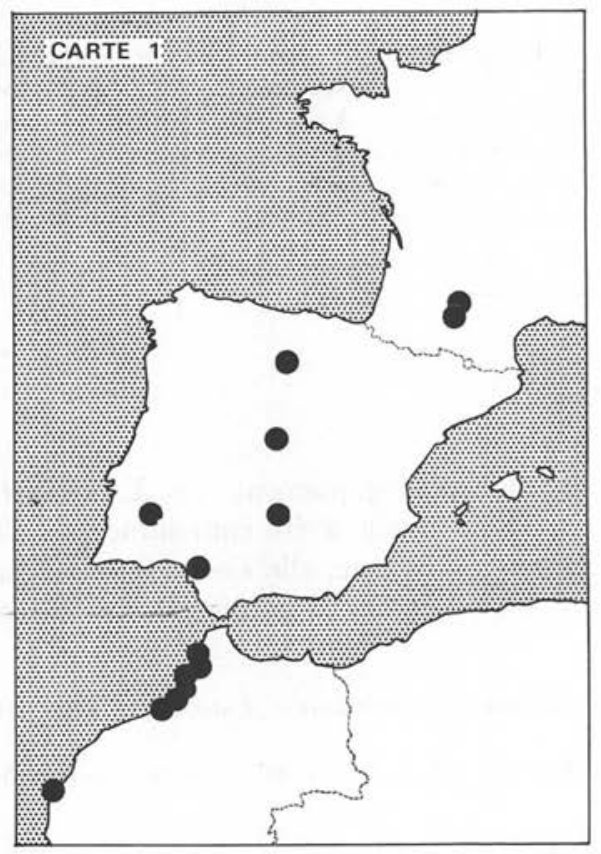

FIG. I. - Xenopsylla cunicularis.

Stations connues dans l'ouest du bassin méditerranéen. 
nom. cheopis, Launay 1981 emend.), Oued Yquem (Province de Rabat), Mehdiya (Province de Kenitra) (Beaucournu et Launay, non publié) et île d'Essaouira (BaillyChoumara rec., Launay 1981, det.).

En ce qui concerne la péninsule ibérique, la répartition de cette espèce y est vaste puisque les cinq stations d'où elle a été signalée se répartissent du Sud au Nord : Coto Doñana, Province de Seville (Espagne) (Lane, 1966), Santa Eulalia, Alto Alentejo (Portugal) (Abreu, 1980), Herencia, Province de Ciudad Real (Espagne) (Rodriguez, 1980), environs de Madrid (Espagne) (Cartaña-Castella et Gil Collado, 1934, sub. nom. cheopis, Beaucournu 1976 emend.) et Torrepadierne, Province de Burgos (Espagne) (Beaucournu, 1976).

Enfin elle a été découverte récemment en France, près de Toulouse au Fauga (Beaucournu et Launay, 1977a) et à Portet-sur-Garonne (Haute-Garonne) (Launay, 1981).

Du point de vue paléobiogéographique, Beaucournu (1981) admet que c'est sur la rive européenne de la Méditerranée que s'est effectué le passage de cette Xenopsylla sur le lapin, les Gerbillidae, hôtes primitifs, disparaissant à la suite sans doute d'une modification du climat.

\section{B - Présentation du gîte}

Le gîte où nous avons effectué cette étude est situé au Fauga (Haute-Garonne) ${ }^{1}$, à $30 \mathrm{~km}$ au sud-ouest de Toulouse. Le terrain, sableux et sec, est recouvert de graminées. Quelques dépressions, plus humides (joncs, saules) l'entrecoupent.

La densité en lapins est y très forte : la population en avril-mai 1977 était de l'ordre de 5000 individus pour 55 hectares soit 90/ha. Deux raisons à cela : d'une part la nature alluvionnaire du sol et la présence de buttes artificielles favorisent l'établissement des garennes (fouissement facile, bon drainage), d'autre part la chasse n'y est pas pratiquée.

$\mathrm{Au}$ point de vue climatique la région Midi-Pyrénées se distingue par son régime des pluies : la saison la plus arrosée est le printemps. Les relevés de 1977 dans la zone qui nous intéresse ( $f$ ig. 2) vont dans ce sens : la pluviosité relativement faible (total pour l'année $667 \mathrm{~mm}$ ) s'est concentrée sur les mois de mai, juin, juillet, très humides. Par contre la fin de l'été et l'automne ont été très secs. La moyenne annuelle des températures a été de $13^{\circ} \mathrm{C}$, l'influence océanique se faisant sentir en hiver où les températures ont été clémentes (moyenne pour janvier, février, mars : 8,5; $M: 12,5, m: 4,5)$.

I. Nous remercions vivement Monsieur Cantau, Directeur de France-Gibiers, pour les facilités qu'il nous a données dans la prospection de ce gite, ainsi que la Direction de la Société nationale des poudres et explosifs qui nous a permis l'accès à cette zone réservée. 


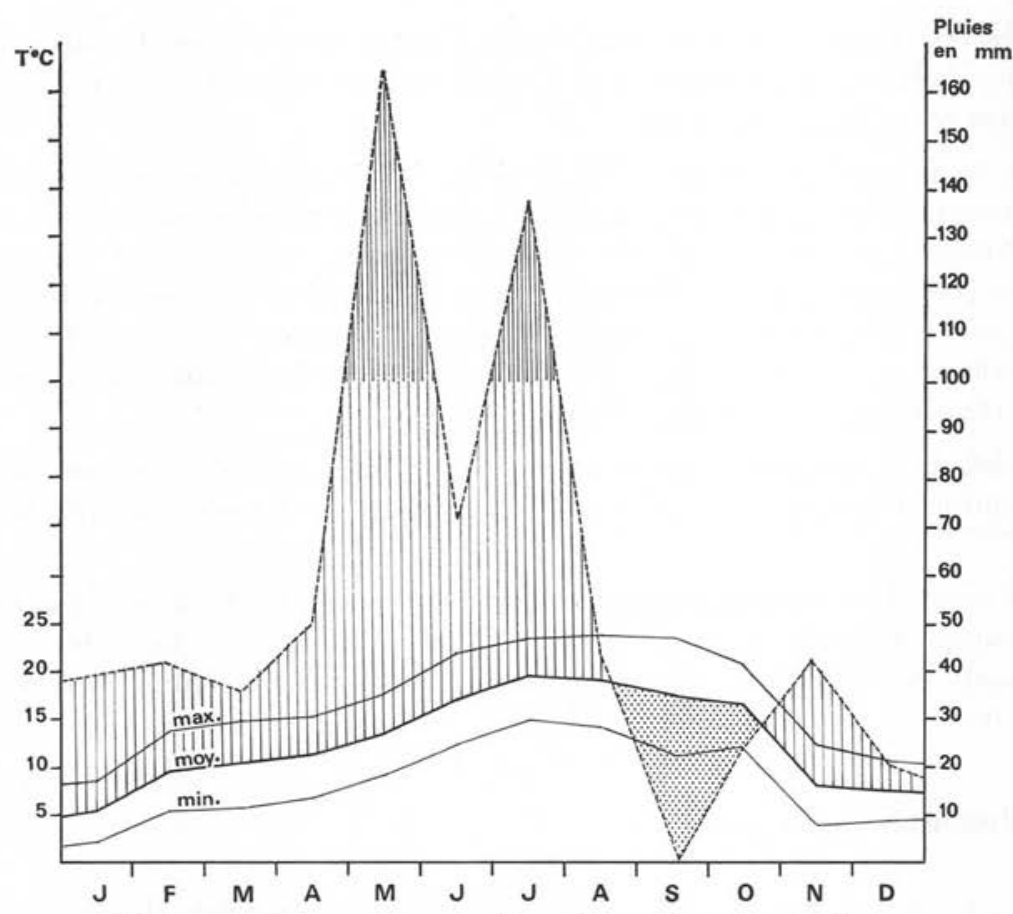

FIG. 2. - Diagramme ombrothermique de la région du Fauga (station météorologique de Cugnaux) pour l'année r977.

\section{C - Techniques de récolte des puces}

\section{1 - Sur les lapins}

Toutes les récoltes de parasites ont été effectuées sur des animaux vivants, relâchés ensuite dans la nature. A cette fin les lapins ont été capturés par furetage. Dès que l'un d'eux se prend dans le filet, il est aussitôt placé au-dessus d'une surface blanche sur laquelle les puces sont récupérées. Une fraction de celles-ci est mise en alcool à $70^{\circ}$, les autres sont ramenées en tube de survie au laboratoire où elles seront disséquées.

\section{2 - Dans les terriers}

Seuls les terriers effectivement habités (traces fraîches, observation directe de l'hôte) ont été retenus pour évaluer les variations de densité des siphonaptères. Par souci d'homogénéisation des résultats, les galeries rectilignes et d'un diamètre régulier (25 à $30 \mathrm{~cm}$ ) ont été préférées aux autres.

Le prélèvement est effectué à l'aide d'une raclette du type décrit par Chiranovitch et coll. (1950), adaptée au diamètre et à la longueur des cavités étudiées.

Le sédiment est recueilli dans une cuvette dans laquelle les puces sont capturées à vue à l'aide d'un aspirateur à bouche. 


\section{II — Résultats}

\section{A - Spécificité parasitaire}

Sur les 109 lapins capturés de janvier 1977 à février 1978 et dans leurs terriers, nous avons recueilli $1480 \mathrm{X}$. cunicularis (428 mâles, 1052 femelles), 2660 S. cuniculi (1 183 mâles et 1477 femelles) mais aucune autre puce, spécifique ou accidentelle.

$X$. cunicularis n'a encore été capturée sur aucun autre mammifère que le lapin (environ 3000 puces de micromammifères ibériques piégées dans des zones où le lapin est très certainement parasité par $X$. cunicularis n'incluaient pas cette espèce). Il semble donc bien qu'elle soit, comme $S$. cuniculi, un parasite spécifique très strict.

\section{B - Les lapins étudiés}

Les premières lapines gestantes sont apparues, en 1977, dès fin janvier et les premiers jeunes dès février ( fig. 3). Le maximum de jeunes a été observé début mai, ce qui indique un pic dans la reproduction début avril. La myxomatose est apparue à la mi-juin et l'épizootie s'est étendue très rapidement et a été particulièrement meurtrière mais brève. Elle a cessé en août ; en septembre nous avons repris quelques individus guéris présentant de fortes cicatrices.

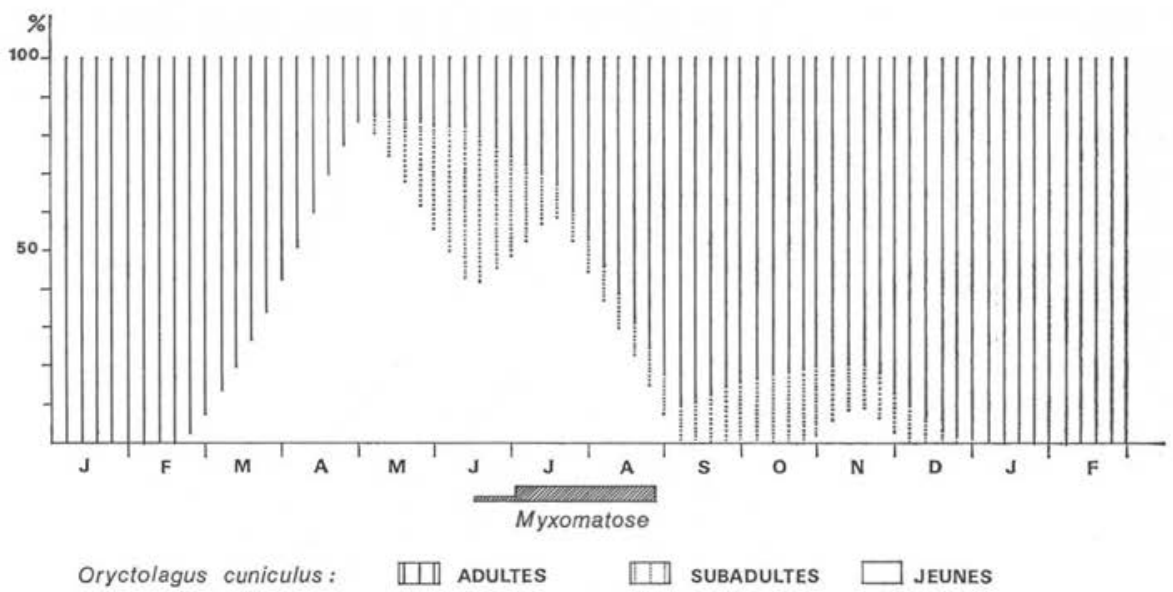

FIG. 3. - Importance relative des différentes classes d'âge (adultes, subadultes, jeunes) d'Oryctolagus cuniculus, dans la station d'étude, au cours de l'année I977.

L'apparition de la myxomatose en juin a marqué l'arrêt brutal de la reproduction. D'autre part la figure 3 montre que la classe d'âge subadulte a été très touchée par la maladie.

Les jeunes capturés en novembre indiquent qu'il y a eu une reprise partielle de la reproduction en octobre, après l'épizootie de myxomatose. 


\section{C - Comportement et localisation de $X$. cunicularis}

\section{1 - Variations saisonnières}

Contrairement à Spilopsylhs cuniculi, X. cunicularis se comporte alternativement comme puce de fourrure ou puce de terrier.

Pendant les mois froids, $X$. cunicularis est rare, voire absente sur le sol des terriers, l'essentiel des prises a été effectué sur les lapins (indice 13,4 en janvier 1977).

Durant les mois où elle a été particulièrement dense (mars à juillet 1977) il est évident qu'elle est proportionnellement beaucoup plus abondante sur le sol des terriers que sur l'hôte. En juillet, par exemple, il y a en moyenne $150 \mathrm{X}$. cunicularis par galerie, de l'entrée à $1 \mathrm{~m}$ de profondeur, et 10,5 par lapin. Pour un terrier comprenant cinq ouvertures et occupé par trois lapins (ce qui nous semble un cas moyen dans la zone d'étude) cela représente donc plus de 750 puces sur le sol (cf. ci-dessous) pour 30 puces sur les hôtes fréquentant ce terrier.

\section{2 - Localisation dans les terriers}

La répartition des prises dans les terriers (fig. 4), effectuées par tranches de $25 \mathrm{~cm}$, montre un pic de densité entre 50 et $75 \mathrm{~cm}$ de profondeur, du moins pour les premiers 100 centimètres à partir de la bouche et entre $11 \mathrm{~h}$. et $18 \mathrm{~h}$. Dans la grande majorité des cas la galerie présente un coude ou bifurque au-delà d'un mètre, il devient donc très difficile d'y comptabiliser les puces. Nous avons cependant pu constater, lorsque cela était possible, qu'à partir de $1,20 \mathrm{~m} \mathrm{X}$. cunicularis devient très rare. D'autre part, elle est absente devant l'entrée du terrier ou dans son voisinage immédiat.

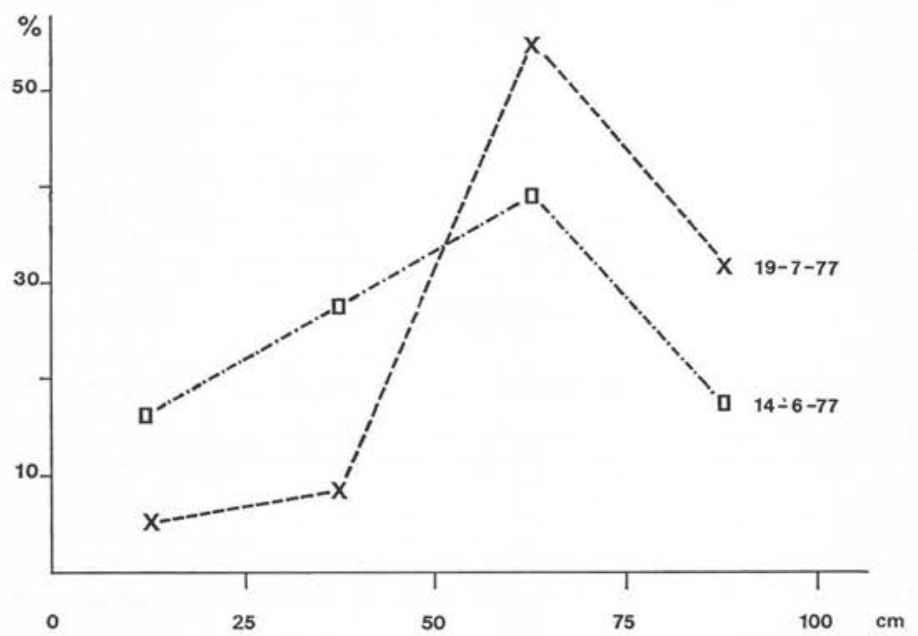

FIg. 4. - Répartition relative des captures de Xenopsylla cunicularis dans les galeries des terriers, par tranche de $25 \mathrm{~cm}$, depuis l'entrée jusqu'à I mètre de profondeur, entre II h. et I8 h., en juin et juillet 1977 . 


\section{3 - Localisation sur l'hôte}

Au Maroc, et d'après Blanc et Bruneau (1956) X. cunicularis se tient sur la face, la poitrine et un peu partout sur le corps. Les exemplaires en provenance d'Espagne ont été capturés par Allan (teste Lane, 1966) sur le museau du lapin. Quant à nous, l'essentiel de nos captures s'est fait sur la partie postérieure de l'animal (dessus et dessous de la queue, arrière-train, face plantaire des pattes) quelque soit le sexe du lapin et la période de l'année. En moyenne, $61 \%$ des $X$. cunicularis se trouvaient au niveau de la queue, $25 \%$ au niveau des pattes arrières et $14 \%$ sur le reste du corps. La figure 5 montre le cas précis de deux lapins : l'un capturé en janvier, l'autre en juillet. Aucune observation de $X$. cunicularis fixée sur les oreilles n'a été faite.

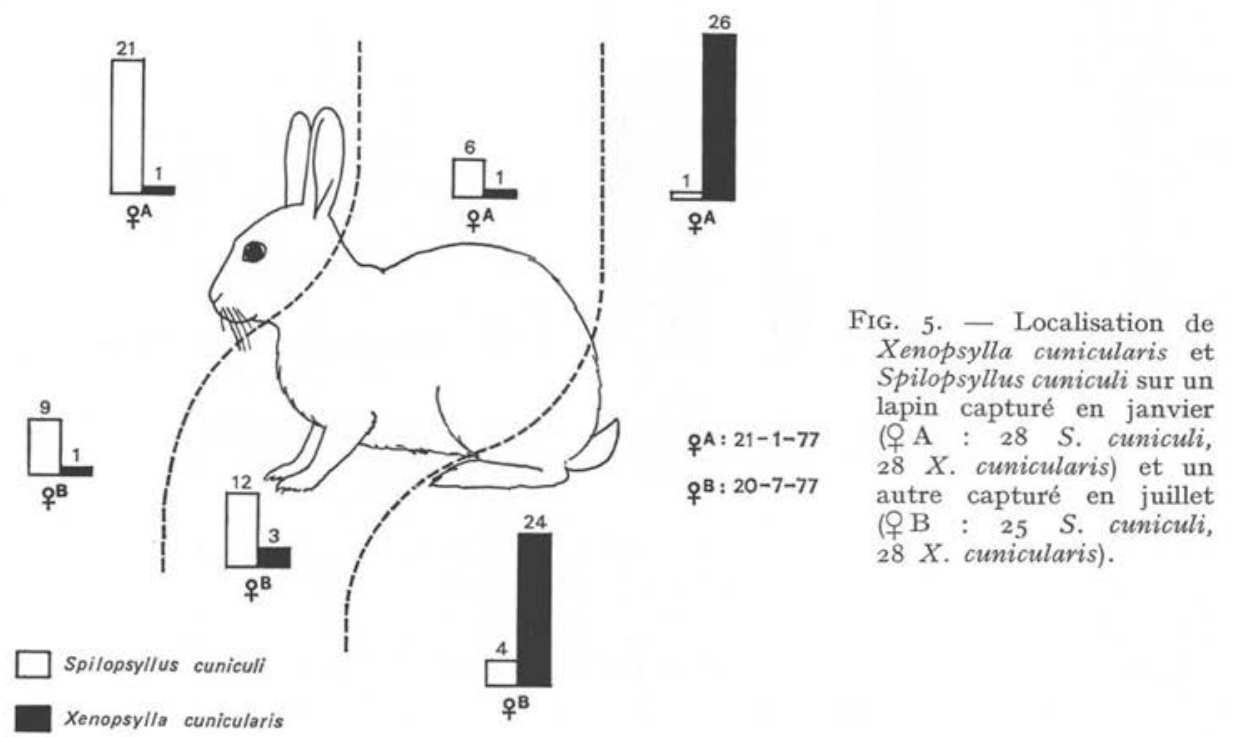

Cette localisation de $X$. cunicularis est originale et il ne semble pas y avoir l'équivalent chez les autres espèces inféodées au lapin de garenne. Ainsi, pendant la majeure partie de l'année, et conformément aux observations d'Allan (1956), S. cuniculi se trouvait cantonnée sur la tête et les oreilles : s'agit-il d'un phénomène de compétition entre les deux espèces ?

\section{D - Variations de l'abondance des imagos}

1 - Dans les terriers (fig. 6)

Dans les galeries la population imaginale était extrêmement réduite, voire absente pendant la période froide, tandis qu'au printemps apparaissait un pic d'abondance (104 exemplaires en moyenne pour le premier mètre de galerie en mars 1977) suivi d'un deuxième en été (152 en juillet 1977). 


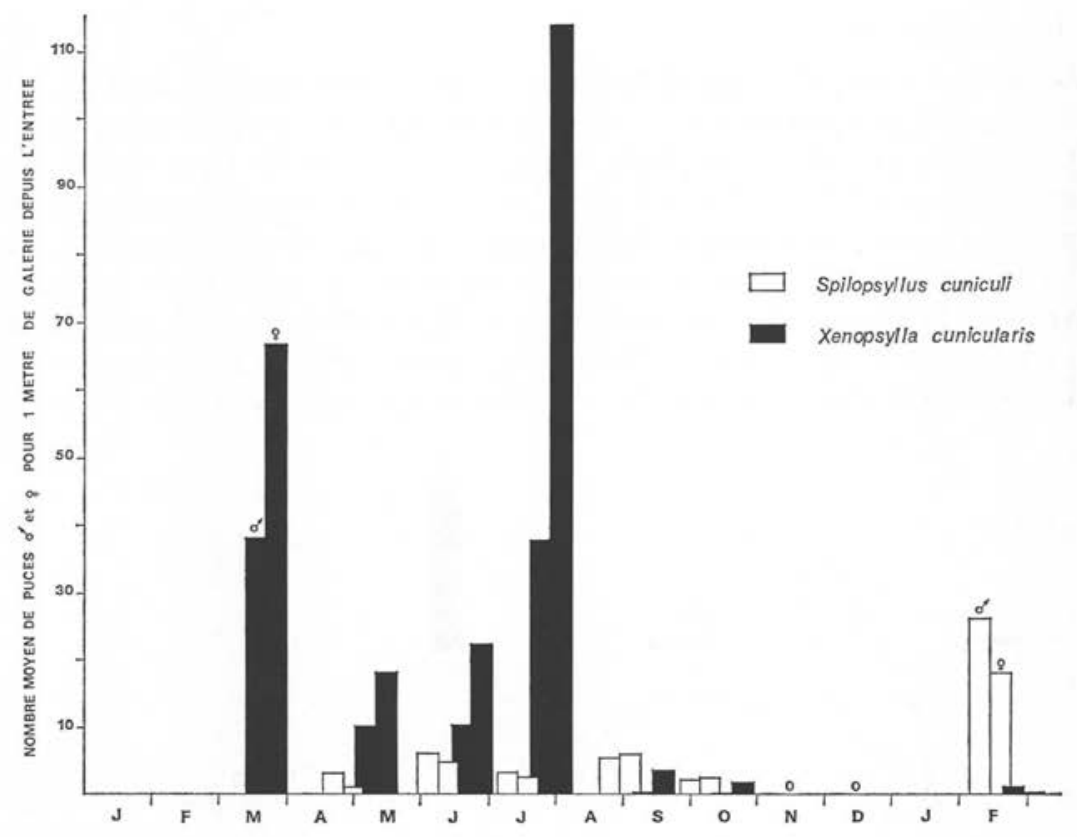

FIG. 6. - Variation annuelle d'abondance de X. cunicularis et S. cuniculi sur le sol des terriers pour un mètre de galerie depuis l'entrée.

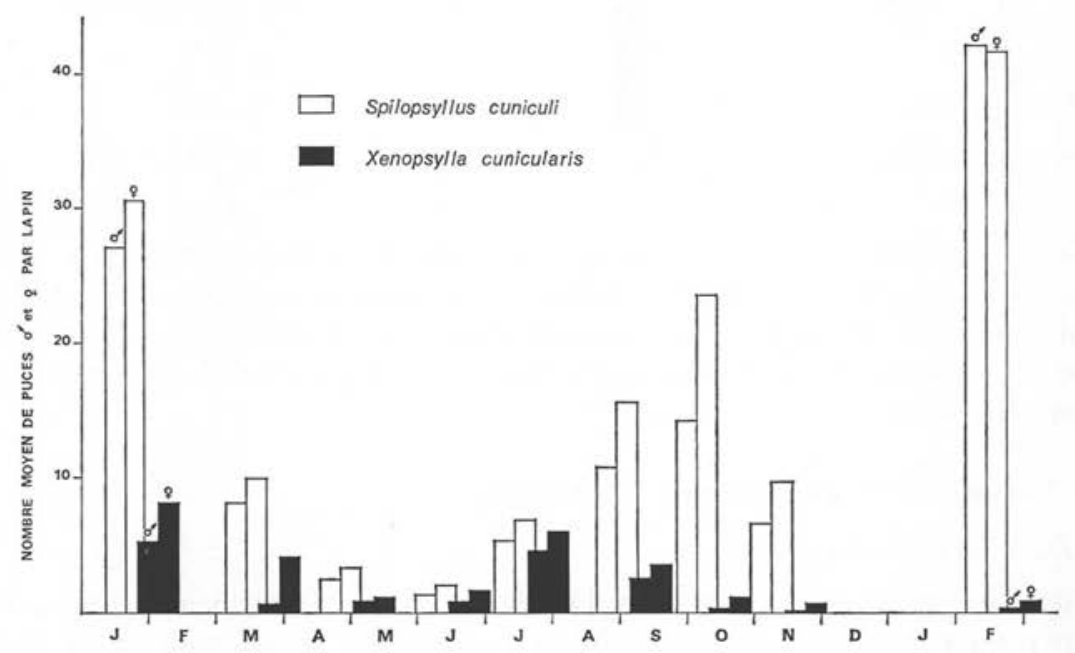

FIg. 7. - Variation de l'indice cunicularis et cuniculi sur $O$. cuniculus au cours de l'année. 
Du point de vue phénologique et en ce qui concerne les émergences imaginales, $X$. cunicularis se présente ici comme une puce verno-estivale.

\section{2 - Sur les lapins}

a) Indice pulicidien (fig. 7)

S. cuniculi a présenté des variations significatives au cours de l'année et nos observations sont qualitativement en accord avec celles d'Allan (1956), Williams (1973) et Mead-Briggs et coll. (1975) : abondante pendant l'hiver, ses effectifs ont chuté fortement à l'approche de l'été.

Comparativement les variations de $X$. cunicularis paraissent beaucoup moins nettes et l'indice sur l'hôte est toujours resté à un niveau relativement faible.

Selon l'intensité de la mortalité pré-automnale, la population imaginale hivernante est plus ou moins importante : ainsi, l'indice pulicidien sur l'hôte (alors bien représentatif de la densité réelle puisque les Xenopsylla ont pratiquement disparu des galeries) était de 13,4 en janvier 1977 et de 1,2 en février 1978.

Pendant la saison chaude, la grande majorité des Xenopsylla se trouve dans les terriers, mais l'augmentation de la population est telle que l'indice sur l'hôte se maintient (juillet $1977: 10,5)$.

b) Fréquence de l'infestation (fig. 8)

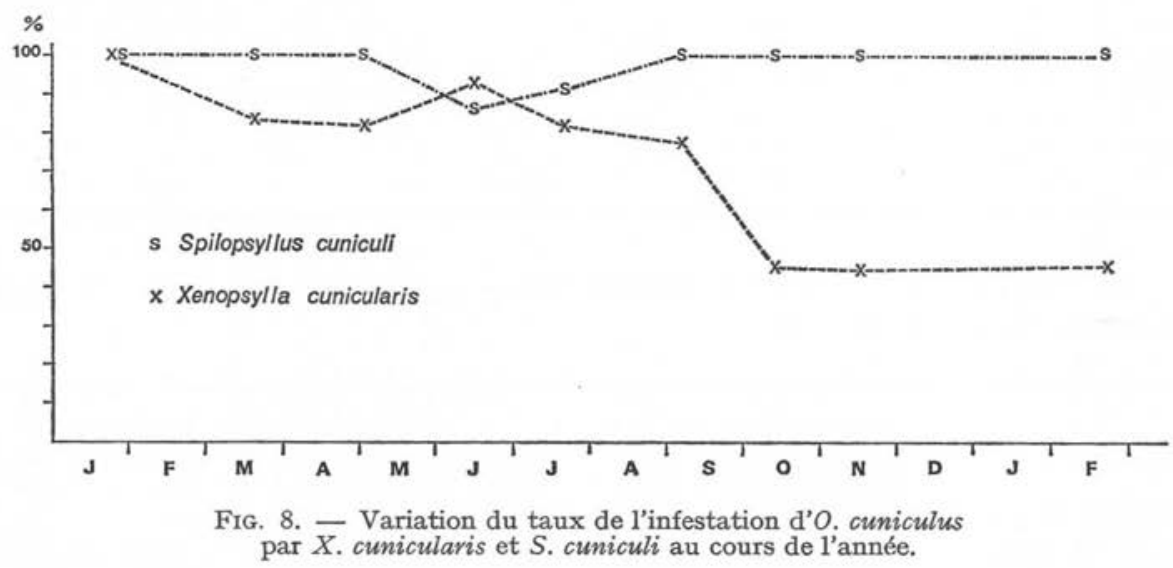

Il apparaît d'emblée un lien étroit entre la fréquence de l'infestation, le niveau de densité de la population pulicidienne et la période de l'année considérée :

- En hiver les $X$. cunicularis sont concentrées sur les lapins. Si l'indice pulicidien est élevé, le taux l'est aussi, les Xenopsylla se distribuent régulièrement : ainsi en janvier 1977 un taux de $100 \%$ était enregistré, qui correspondait à un indice mensuel élevé $(13,4)$. Par contre, pendant la même période, si l'indice est bas, le taux baisse : avec un indice très faible de 1 en novembre 1977, le nombre de lapins parasités tombe en dessous de $50 \%(44,4)$ en février 1978 : indice 1,2 , taux $45 \%$. 
- Au printemps et en été l'infestation des lapins, d'un individu à un autre, devient irrégulière. Le comportement de $X$. cunicularis a changé : elle se trouve essentiellement dans les terriers où elle copule et pond, elle reste peu sur son hôte (probablement le temps de se gorger). Le comportement des lapins intervient aussi : certains individus fréquentent davantage les terriers que d'autres, ils ont donc plus de risques d'être parasités. Enfin toutes les garennes ne présentent pas la même densité en Xenopsylla, certaines offrant des microconditions défavorables (en particulier sol trop humide, gorgé d'eau) seront plus pauvres en Xenopsylla et verront par conséquent leurs habitants peu parasités. Ainsi, pendant tous ces mois où Xenopsylla est abondante, le taux de parasitisme est inférieur à 100\% (mars 1977: $88 \%$, juillet : $82 \%$ ).

c) Influence du sexe et de l'âge de l'hôte

Nous n'avons pas pu mettre en évidence des différences significatives dans le nombre des $X$. cunicularis sur les lapins en fonction de l'âge et du sexe de ceux-ci, contrairement à $S$. cuniculi à certaines périodes de l'année.

Ainsi, de mars à juillet 1977, les indices pulicidiens pour les différentes classes d'âge des lapins ont été les suivants :

\begin{tabular}{|c|c|c|c|c|c|c|c|c|c|}
\hline & & \multicolumn{2}{|c|}{ Mars } & \multicolumn{2}{|c|}{ Mai } & \multicolumn{2}{|c|}{ Juin } & \multicolumn{2}{|c|}{ Juillet 1977} \\
\hline & & X.c. & S.c. & X.c. & S.c. & X.c. & S.c. & X.c. & S.c. \\
\hline \multirow[b]{2}{*}{$\mathrm{P} / \mathrm{H}$} & Jeunes & 2,5 & $2,3 *$ & 2,1 & $5,9 *$ & 2,0 & 5,3 & 6,7 & 8,7 \\
\hline & $\begin{array}{l}\text { Subadultes } \\
\text { Adultes }\end{array}$ & $\overline{6,0}$ & $\overrightarrow{42,0} *$ & $\overline{1,5}$ & $1 \overline{27,0}$ * & $\begin{array}{l}2,7 \\
2,6\end{array}$ & $\begin{array}{l}1,8 \\
5,3\end{array}$ & $\overline{16,0}$ & $\overline{18,0}$ \\
\hline
\end{tabular}

De septembre 1977 à février 1978 les indices pulicidiens, en fonction du sexe des lapins ont été les suivants :

\begin{tabular}{|c|c|c|c|c|c|c|c|c|c|}
\hline & & \multicolumn{2}{|c|}{ Septembre } & \multicolumn{2}{|c|}{ Octobre } & \multicolumn{2}{|c|}{ Novembre 1977} & \multicolumn{2}{|c|}{ Février 1978} \\
\hline & & X.c. & S.c. & X.c. & S.c. & X.c. & S.c. & X.c. & S:c. \\
\hline $\mathrm{P} / \mathrm{H}$ & $\begin{array}{l}\hat{\delta} \text { adultes } \\
\text { adultes }\end{array}$ & $\begin{array}{l}4,0 \\
8,6\end{array}$ & $\begin{array}{l}23,5 \\
28,8\end{array}$ & $\begin{array}{l}3,0 \\
0,9\end{array}$ & $\begin{array}{l}40,0^{*} \\
19,6^{*}\end{array}$ & $\begin{array}{l}2,0 \\
0,6\end{array}$ & $\begin{array}{l}28,5^{*} \\
15,2^{*}\end{array}$ & $\begin{array}{l}1,4 \\
1,0\end{array}$ & $\begin{array}{l}58,8^{*} \\
109,4^{*}\end{array}$ \\
\hline
\end{tabular}

\section{3 - Le sex-ratio (fig. 6 et 7 )}

Les deux espèces ont un sex-ratio en faveur des femelles toute l'année. S. cuniculi présente une moyenne annuelle de 0,80 ; chez $X$. cunicularis la prépondérance des

\footnotetext{
variance.

* Indices significativement différents à un niveau de certitude de $99 \%$ par analyse de la
} 
femelles est encore plus accentuée puisque cette moyenne descend à 0,41 (chiffres établis à partir de l'ensemble des prises hôtes et terriers). C'est en juillet, fin de la période d'émergence dans nos relevés, que $X$. cunicularis a présenté le plus fort déséquilibre dans le sex-ratio (fig.7), soit 0,35 (74\% de femelles). Une explication possible à ce déséquilibre qui s'accentue pendant la période d'activité sexuelle est que les mâles subissent une mortalité plus grande que celle des femelles.

\section{E - Structure de la population imaginale de $X$. cunicularis par groupes d'âge physiologique}

\section{1 - Mâles}

Nous regroupons les mâles en deux catégories :

- groupe I : les néonates ou immatures : l'estomac contient du méconium bien visible par transparence. Nous ajoutons à cette catégorie les " jeunes ", qui ont déjà pris un repas de sang, mais qui sont encore très peu sclérifiés, avec une plaque pénienne claire.

Des néonates ont été observés en mars (fig. 9) conjointement à l'augmentation de population de printemps, puis en juillet qui correspondait au deuxième pic d'émergence, puis encore en septembre.

- groupe II : les âgés ou matures : les génitalia sont bien sclérifiés, brunâtres, le tégument, surtout au niveau des tergites abdominaux, relativement foncé.

Dans ce groupe, selon l'état physiologique, nous séparons les mâles aux antennes dressées des autres.

Le redressement des antennes chez le mâle prend place dans la séquence de l'acte copulatoire, sa signification est discutée ( $c f$. en particulier Holland 1955, Humphries 1967). Nous n'avons trouvé dans la littérature aucune indication sur la durée de ce phénomène : existe-t-il une remanence plus ou moins prolongée, même en l'absence de femelles?

Chez X. cunicularis il semble bien que cette érection antennaire, une fois initiée, persiste jusqu'à la fin de la période de reproduction, donc entre les copulations.

Ceci nous paraît intéressant car dans l'hypothèse où cette manifestation traduit une activité sexuelle, la proportion de mâles aux antennes dressées reflète le degré d'intensité de la reproduction dans la population.

Les premiers mâles à antennes dressées (observation in vivo) sont apparus en mars, leur nombre n'a ensuite cesser d'augmenter jusqu'en septembre où il atteignait $100 \%$ (néonates non compris) pour tomber ensuite très rapidement.

\section{2 - Femelles}

Les femelles ont été disséquées selon la technique exposée par Mead-Briggs (1962), dans de l'alcool à $30 \%$.

Prokopiev (1958), Kunitskaya (1960), Klein (1966), Klein et coll. (1973) classent les femelles en cinq groupes d'âge physiologique selon l'activité de ponte passée : néonates, nullipares nourries, unipares, paucipares, multipares. Dans des conditions écologiques favorables, la majorité des femelles des espèces concernées suivent cette 


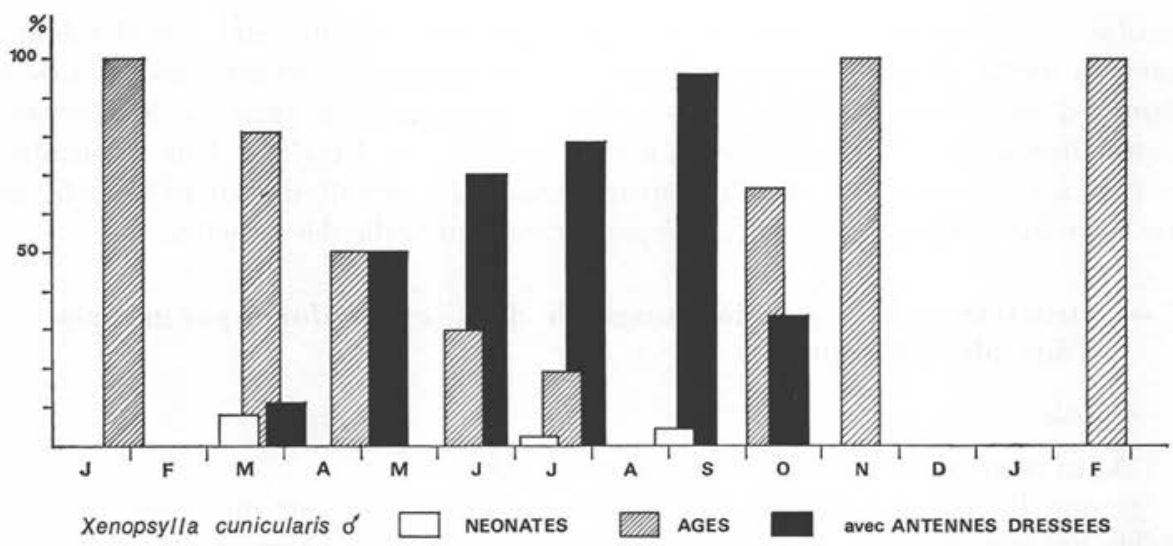

FIG. 9. - Évolution annuelle de l'importance relative des groupes d'âge physiologique (voir définition dans le texte) chez les mâles de X. cunicularis.

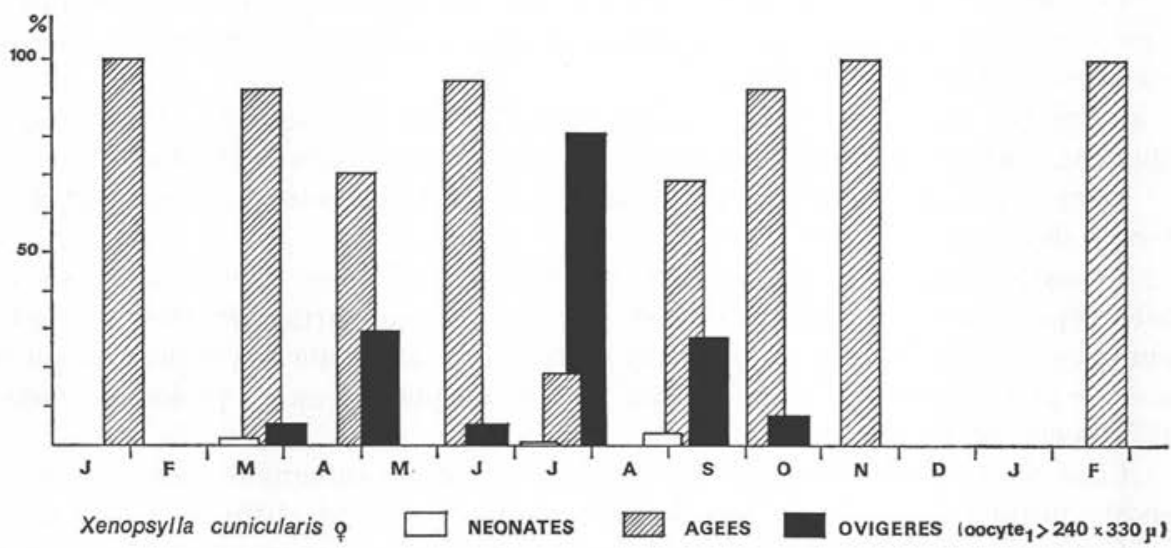

FIg. ro. - Évolution annuelle de l'importance relative des groupes d'âge physiologique (voir définition dans le texte) chez les femelles de $X$. cunicularis.

séquence et passent par tous les stades dans un laps de temps court. Kunitskaya et coll. (1977) proposent un $6^{\mathrm{e}}$ groupe en envisageant le cas des femelles nées en fin de période de reproduction et qui n'amorcent pas dans l'immédiat une maturation oocytaire : le stade nullipare nourri est scindé en deux, gonoactives ou non.

En ce qui concerne les femelles de $X$. cunicularis, des difficultés sont apparues pour apprécier la formation et le nombre des corps jaunes. Dans de nombreux cas, cela était réalisable, mais ne réflétait pas réellement l'âge de l'individu, car un blocage de l'activité sexuelle est possible pour tous les stades. Ainsi une femelle paucipare, voire nullipare pourrait déjà avoir plusieurs mois d'existence. Ces considérations n'interviennent pas pour délimiter dans le temps la période de reproduction. 
Pour ces raisons nous avons adopté une classification simplifiée, basée non plus sur l'activité de ponte passée (quantité d'œufs pondus) mais sur l'activité gonadique présente, soit schématiquement : les femelles ayant des œufs mûrs, prêts à la ponte, et les autres. Parmi ces dernières, les néonates se reconnaissent facilement, aussi nous les classerons à part.

Nous distinguerons donc :

- groupe I : les femelles néonates, Les oocytes 1 (4 à 6 ovarioles chez X. cunicularis) sont en phase 2-3 (Kunitzkaya, 1960) avec un diamètre de 100 à $130 \mu \mathrm{m}$. Du méconium est présent dans l'intestin, la bulga de la spermathèque est brun-clair. Celle-ci reste claire en l'absence de repas de sang, mais les téguments se sclérifient avec le temps.

Comme pour les mâles, les femelles néonates étaient présentes dans nos prélèvements en mars, juillet et septembre ( $f$ ig. 10).

- groupe II : femelles nourries, bulga marron foncé. Ce caractère de pigmentation de la spermathèque est largement utilisé pour distinguer les jeunes femelles des adultes dans le genre Xenopsylla. L'obscurcissement intervient en quelques jours et est d'autant plus rapide que la température est élevée (Gerasimova, 1971). Les oocytes 1 sont en phase 3 à 5 (Kunitzkaya, 1960) avec un diamètre de 130 à $330 \mu \mathrm{m}$. Comme le souligne Mead-Briggs (1977) à propos de S. cuniculi, ces oocytes sont, pour les plus petits, soit en phase initiale de maturation, soit en phase finale de régression et pour les plus gros, soit en phase de dépôt rapide de vitellus et maturation, soit en phase de résorption et régression. Il est donc difficile de dire si ces femelles sont physiologiquement gonoactives, gonodépressives ou gonoinactives.

- groupe III : ce sont les femelles ovigères. L'oocyte 1 a une taille supérieure à $240 \times 330 \mu \mathrm{m}$ (phase 6 de Kunitzkaya), l'oviposition est inéluctable et imminente.

Il y avait des femelles ovigères de mars à octobre, le pic se situant en juillet. Ceci concorde également avec la période où les mâles ont les antennes dressées.

Proportionnellement, les femelles ovigères étaient plus nombreuses sur le sol des terriers que sur les lapins (respectivement $81,1 \%$ et $3 \%$ en juillet).

La majorité des femelles hivernantes présentaient des ovaires réduits et n'avaient pas de corps jaunes visibles. Elles appartenaient vraisemblablement à la dernière génération de l'année, émergées en fin de période de reproduction (néonates de septembre) et au moment où la population subissait une forte mortalité.

\section{III - Discussion}

\section{A - Données écologiques sur les Xenopsylla du groupe conformis}

$X$. cunicularis est étroitement apparentée aux Xenopsylla du groupe conformis qui renferme un ensemble d'espèces très proches non seulement sur le plan morphologique, mais aussi écologique. Essentiellement parasites de Gerbillidés (sauf cunicularis), elles ont été très étudiées par les auteurs soviétiques car elles tiennent une place importante dans l'épidémiologie de la peste. 
Le cycle annuel de $X$. skrjabini, parasite de Rhombomys opimus, se déroule, dans la région nord-est de la Caspienne, de la façon suivante (Gerasimova 1969b) : $X$. skrjabini passe l'hiver sous forme adulte et reprend son activité reproductrice à partir d'avril. Le nombre maximum de femelles en oviposition se situe en avril-mai, celles-ci sont peu nombreuses en septembre, et en octobre toute activité de ponte disparait. Les photopériodes longues favorisent la maturation et la fécondité des femelles. Le maximum d'émergences a lieu en juin-juillet, mais la population est la plus élevée en octobre. Il faut noter que les adultes de la génération d'été ont une vie courte. $X$. skrjabini présente dans ces conditions, deux générations par an, le renouvellement ayant lieu en juin-juillet et octobre-novembre.

Les pontes et les développements larvaires sont continus (Zolotova et coll., 1978) avec par conséquent un chevauchement des générations qui peuvent atteindre quatre dans l'année, la population hivernante imaginale incluant les individus des $2^{\mathrm{e}}, 3^{\mathrm{e}}$ et $4^{\mathrm{e}}$ générations. Yakunin et coll. (1979) trouvent également 4 générations annuelles pour $X$. skrjabini dans le Mangyshlak, les $3^{\mathrm{e}}$ et $4^{\mathrm{e}}$ générations hivernent et commencent la reproduction au printemps de l'année suivante pour donner départ en juin à la $1^{\text {re }}$ génération d'été. Le cas général est que les dernières générations de l'année, c'est-à-dire celles qui vont passer l'hiver, restent immatures jusqu'au printemps suivant.

La plupart des auteurs s'accordent à dire que la forme hivernante est l'adulte seul et que c'est ce stock qui, devenant actif sexuellement au printemps suivant, met en place la nouvelle génération. Ainsi Kir'Yakova et coll. (1970) signalent à ce sujet que ni larves, ni pupes ne sont trouvées dans les nids de Rhombomys durant la fin de l'automne, l'hiver ou en mars. Il semble de toute façon que ces Xenopsylla ne fréquentent ni les parties profondes des terriers, ni les nids, d'une manière générale et que la ponte et le développement larvaire se déroulent dans les galeries (Bibikova et coll., 1963). Klein (1963) note également que $X$. buxtoni est rare dans les litières de Mérions malgré son extraordinaire abondance dans les galeries des terriers. Dans certains cas particuliers toutefois, la forme hivernante peut être un autre stade que l'adulte ; en effet Darskaya (1955) signale que la dernière génération peut être active sexuellement juste avant la saison défavorable et que les œufs alors pondus ne donnent pas d'adultes avant l'année suivante.

Un aspect fondamental de l'écologie des Xenopsylla du groupe conformis est la présence et l'importance de leurs effectifs dans les galeries des terriers de Gerbilles, pendant la période d'abondance. Ces effectifs sont constitués d'une part de néonates qui ont tendance à migrer vers les parties hautes (recherche de l'hôte), d'autre part de puces en activité sexuelle (copulation, ponte) (Bibikova et coll., 1965). Le dénombrement dans les terriers est donc essentiel pour apprécier de façon réaliste le niveau de population.

Température et humidité agissent différemment sur le développement des stades larvaires (Gerasimova, 1969a), sur la fécondité (Zolotova et Afanas'eva, 1969) et la survie des adultes (Gerasimova et Gavrikova, 1970) et il apparaît dans tous les cas une autorégulation des populations des Xenopsylla du groupe conformis essentiellement liée aux facteurs abiotiques : l'élévation de température déclenche 
la maturation sexuelle, accélère la production d'œufs, favorise le développement larvaire mais en revanche entraîne une forte mortalité des adultes ; les basses températures bloquent la reproduction mais en compensation offrent aux imagos un taux de survie considérable qui permet à la population de se maintenir à un niveau correct.

\section{B - Ébauche du cycle annuel de $X$. cunicularis}

$X$. cunicularis, malgré sa spécificité très différente, a gardé sur le plan écologique les caractéristiques du groupe conformis. L'apparition de la nouvelle génération d'adultes a lieu au début du printemps : néonates ( fig. 9 et 10) et premier pic d'abondance (fig. 4) en mars. Nous pensons que cette nouvelle génération a été mise en place en partie à l'automne précédent (donc diapause hivernale, outre la forme adulte, soit au stade œuf, soit au stade nymphe) car l'activité reproductrice était nulle avant ce premier pic d'abondance. La deuxième génération d'adultes se manifeste au début de l'été : néonates ( $f i g .9$ et 10), deuxième pic d'abondance ( $f i g$. 4) encore plus élevé, activité reproductrice intense (maximum annuel de femelles ovigères) en corrélation avec l'élévation de température et une forte hygrométrie. La troisième génération d'adultes n'est mise en évidence à la fin de l'été que par la présence de néonates; la population a subi une mortalité considérable liée elle aussi aux températures chaudes, et à l'approche de l'automne, à une pluviométrie très faible qui a entrainé une période de forte sécheresse (fig. 2).

Parallèlement à cette mortalité, les signes de reproduction diminuent pour disparaître complètement à l'approche de l'hiver.

A cette phénologie se juxtapose un cycle comportemental : les $X$. cunicularis passent l'hiver, en repos sexuel, sur l'hôte, tandis qu'à la saison favorable elles se trouvent essentiellement sur le sol des terriers. Blanc et Bruneau (1956), lors d'une étude effectuée au Maroc, dans la forêt de Nefifikh, avaient déjà noté la présence de $X$. cunicularis dans les terriers mais avec une phénologie de type hivernal ; les imagos seraient très abondants pendant l'hiver, ils deviendraient rares l'été après une chute brutale de leurs effectifs au printemps. Il ressort de ceci que ce cycle est totalement inversé par rapport à ce que nous avons observé dans le Sud-Ouest ou par rapport aux données des auteurs russes pour les Xenopsylla du groupe conformis; cela s'explique peut-être par une climatologie particulière sur la côte marocaine lors de l'étude (hiver doux et pluvieux, printemps et été très secs), mais mériterait confirmation.

Les données recueillies sur la Péninsule Ibérique font de $X$. cunicularis une puce plutôt estivo-automnale. C'est en septembre-octobre que Abreu (1980) trouve le plus de Xenopsylla sur les lapins (indice de l'ordre de 3) dans la région du Haut Alantejo (Portugal) et Rodriguez (1980) de juillet à septembre dans la région de Ciudad Real (Espagne) avec un indice maximum de 2 en septembre. Dans ces deux stations Xenopsylla devient rare, voire absente sur les lapins pendant le reste de l'année. Il faut toutefois signaler que ces deux études n'envisagent pas les Xenopsylla se trouvant dans les terriers. 


\section{C - Relations gonadotrophiques hôte/parasite}

Plusieurs Spilopsyllinae (Pulicidae) parasites de Leporidae sont liés au cycle gonadotrophique de leur hôte : Spilopsyllus cuniculi sur le lapin (Mead-Briggs, 1964), Cediopsylla simplex sur Sylvilagus floridanus (Rothschild et Ford, 1972) et Cediopsylla tepolita sur Romerolagus diazi (Barrera, 1966). Par contre Graves et coll. (1978) suggèrent qu'un autre Spilopsyllinae, Euhoplopsyllus glacialis affinis, n'est pas lié au cycle gonadotrophique de son hôte (dans cette étude, Sylvilagus audubonii) et les données que nous avons pu recueillir en France sur cette puce (sur Sylvilagus floridanus) vont dans le même sens (Launay, 1981).

En ce qui concerne $X$. cunicularis (Pulicidae, Xenopsyllinae), nous ne pensons pas non plus que son cycle de reproduction soit lié à celui du lapin. En effet les observations de terrain montrent, d'une part, que le maximum d'activité sexuelle de cette puce (juillet, août) présente un décalage important avec celui de son hôte (mars à mai) ; il n'y avait d'ailleurs plus aucune lapine gestante pendant les mois d'été en 1977 au Fauga. D'autre part, nous l'avons déjà souligné, il n'y avait pas de corrélations entre l'indice cunicularis et le sexe ou l'âge des lapins. Enfin, au laboratoire, lors d'essais d'élevage, nous avons obtenu de nombreuses larves de $X$. cunicularis à partir d'adultes nourris sur lapines non gestantes. Rothschild et coll. (1970) ont montré, d'ailleurs, qu'une autre Xenopsyllinae, $X$. cheopis n'est pas liée non plus au cycle gonadotrophique de son hôte. Pour les Xenopsylla du groupe conformis, auxquelles appartient cunicularis, rien n'est connu ou signalé dans la littérature à ce sujet.

\section{D - Comparaison avec une autre puce spécifique du lapin de garenne : Spilopsyllus cuniculi}

$X$. cunicularis et $S$. cuniculi sont toutes deux des parasites spécifiques du lapin de garenne. Cependant cette spécificité revêt des caractères différents que nous résumons dans le tableau $I$ :

S. cuniculi présente des liens extrêmement étroits avec le lapin, elle est intimement adaptée à son hôte, ce qui lui permet d'une certaine façon d'échapper aux conditions extérieures. La phase critique de son cycle, le développement larvaire, a lieu dans les conditions les plus favorables, c'est-à-dire dans ce microcosme que constitue la rabouillère ${ }^{1}$. Ceci est possible grâce au couplage parfait entre les cycles gonadotrophiques de la puce et de son hôte. En contrepartie la productivité de $S$. cuniculi est modérée : 1 génération annuelle, fécondité faible.

Les relations $X$. cunicularis-lapin paraissent beaucoup moins sophistiquées et la spécificité de Xenopsylla semble être au premier abord une spécificité écologique : elle est liée aux terriers de lapins où existe une certaine thermohygrorégulation dont le niveau dépend du climat général de la région, elle est donc liée aussi au climat, enfin elle est liée à un certain type de substrat. Cette dépendance vis-à-vis des conditions extérieures est compensée par une forte productivité : natalité élevée, plusieurs

I. La rabouillère est le trou creusé par la femelle gestante, garni d'herbes sèches et de poils, où elle mettra bas. 
TABLEAU I. - Comparaison des principales caractéristiques écologiques de Xenopsylla cunicularis et Spilopsyllus cuniculi.

\begin{tabular}{|c|c|c|}
\hline & X. cunicularis & S. cuniculi \\
\hline Spécificité & Stricte : O. cuniculus & Stricte : O. cuniculus \\
\hline Répartition & $\begin{array}{l}\text { limitée : S O de la France } \\
\text { Péninsule ibérique, Maroc }\end{array}$ & celle de son hôte \\
\hline $\begin{array}{l}\text { Liens avec les facteurs } \\
\text { abiotiques }\end{array}$ & $\begin{array}{l}\text { climat humide et chaud de type } \\
\text { océanique avec les pluies au } \\
\text { printemps; terrains sableux } \\
\text { et alluvionnaires ; terriers de lapin }\end{array}$ & relative indépendance \\
\hline $\begin{array}{l}\text { Lié au cycle gonado- } \\
\text { trophique de l'hôte }\end{array}$ & 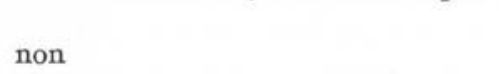 & oui \\
\hline Période de reproduction & Mars à octobre & $\begin{array}{l}\text { celle de son hôte } \\
\text { (février à juillet) }\end{array}$ \\
\hline Développement larvaire & dans les terriers & dans les rabouillères \\
\hline $\begin{array}{l}\text { Nombre de générations } \\
\text { par an }\end{array}$ & 1 à $3-4$ & 1 \\
\hline Émergences d'adultes & mars à septembre & août-septembre à février \\
\hline Abondance & mars à août & septembre à avril \\
\hline Comportement & $\begin{array}{l}\text { puce de fourrure, automne-hiver } \\
\text { puce de terrier, printemps-été }\end{array}$ & puce de fourrure \\
\hline $\begin{array}{l}\text { Localisation } \\
\text { sur le lapin }\end{array}$ & $\begin{array}{l}\text { arrière-train, queue, } \\
\text { pattes arrières }\end{array}$ & tête, oreilles \\
\hline
\end{tabular}

générations annuelles. Cependant cette spécificité est stricte et $X$. cunicularis présente des adaptations morphologiques caractéristiques des puces de Leporidae (Launay et Beaucournu, en préparation), ce qui lui permet sans doute de survivre pendant l'hiver car elle reste alors dans la fourrure de son hôte.

\section{E - Importance épidémiologique}

$X$. cunicularis est très abondante de mars à août et elle peut alors jouer un rôle très actif dans la vection du virus myxomateux dans la mesure où davantage puce de terrier que de fourrure, elle change d'hôte à chaque repas sanguin. Ceci peut avoir des conséquences épidémiologiques certaines car, là où cohabitent $S$. cuniculi et $X$. cunicularis, une couverture parasitaire importante est assurée pendant toute l'année, Xenopsylla prenant au printemps le relais de Spilopsyllus qui a assuré l'entretien hiverno-vernal du virus, et intervenant alors dans les épizooties vernoestivales. Il est d'ailleurs intéressant de constater que dans la zone d'étude la myxomatose sévissait intensivement de juin à août.

D'autre part, divers autres zoonoses à transmission par arthropodes se rencontrent spontanément chez le lapin de garenne (Beaucournu et Launay, 1977b) et ceci particulièrement en zone méditerranéenne. Il n'est pas douteux que $X$. cunicularis puisse intervenir dans ces affections et à ce titre mérite toute notre attention. 


\section{BIBLIOGRAPHIE}

ABREU M. H. : Quelques aspects particuliers du cycle annuel d'infestation du lapin de garenne par deux espèces de puces. Proc. rth Intern. Conf. on Fleas, Ashton Wold, England. Balkema (A. A.) Ed., Rotterdam, I980, pp. 391-396.

Allan R. M. : A study of the populations of the rabbit flea Spilopsyllus cuniculi (Dale) on the wild rabbit Oryctolagus cuniculus in north-east Scotland. Proc. R. ent. Soc. Lond. (A), I956, $3 I, 145-\mathrm{I} 52$.

BarRera A. : Redefinicion de Cediopsylla Jordan y Hoplopsyllus Baker. Revta. Soc. mex. Hist. nat., I966, 27, 67-88.

BeAucournu J. C. : Contribution à l'étude des siphonaptères de mammifères du nord-ouest de la région méditerranéenne (France, Italie, Péninsule ibérique). Thèse Doct. État Sciences, 1976, Rennes, 284 p.

Beaucournu J. C. : Les ectoparasites du Lapin de Garenne, Oryctolagus cuniculus : apports à son histoire. Bull. mens. Off. Nation. Chasse, I981, No Sp. Scien. Techn., 23-35.

Beaucournu J. C., Launay H. : Présence en France d'une Xenopsylla selvatique $X$. cunicularis Smit, 1957 (Siphonaptera, Pulicidae) parasite du lapin de garenne. Bull. Soc. Path. exot., r977a, 70, 299-301.

Beaucournu J. C., Launay H. : Le lapin de garenne et les zoonoses. Méd. Mal. Infect., I977b, $I I, 495-501$.

Bibikova V. A., Gorbunova A. I., Maslennikova Z. P., Morozova I. V., Shmuter M. F. : (On the technique of studying population density of fleas of Rhombomys opinus Licht.). Zool. Zh., 1965, 44, 1214-1218.

Bibikova V. A., In'inskaya V. L., Kaluzhenova Z. P., Morozova I. V., Shmuter M. F. : (Biology of fleas of the genus Xenopsylla in Sary-Ishikotrau desert). Zool. Zh., 1963, 42, I045-1051.

Blanc G., Bruneau J. : Étude épidémio-écologique dans la forêt de Nefifik. I - Présence chez le lapin de garenne et ses arthropodes piqueurs de virus pathogènes pour l'homme. Arch. Inst. Pasteur, Maroc, 1956, s, 87-200.

Blanc G., Delage B., Ascione L. : Étude épidémio-écologique dans la forêt du Cherrat. Arch. Inst. Pasteur, Maroc, 1962, 6, 223-292.

Cartana-Castella P., Gil Collado J. : Estudio de las ratas y de sus ectoparasitos en ocasion del brote epidemico de peste en Barcelona en I931. Comision Perm. Iàvest. Sanit., I934, Madrid, I I $\mathrm{p}$.

Chiranovitch P. I., Mironov N. P., Fomitcheva A. S. : (Méthodes de récolte des parasites aptères des terriers de rongeurs.) Isl. Akad. Nauk. SSSR, Moscou, I950, I 2.

DARSKAYA N. F. : (Peculiarities of the Ecology of Xenopsylla gerbilli caspica. I - Fleas of the large Gerbil in relation to the characteristics of the Ecology of their hosts). (Natural Nidi of human Diseases and regional Epidemiology). PAvLOvSKII E. N. et coll. ed.,I955, Leningrad, 4I 5-424,

Gerasimova N. G. : (The effect of temperature and humidity on the development of the immature stages of two species of fleas from the Great Gerbil). Parazitologiya, 1969a, 3, 24-33.

Gerasimova N. G. : (On the annual development cycle of the flea Xenopsylla skrjabini.). Zool. Zh., I969b, 48, I4IO-I4I2.

Gerasimova N. G. : (The maturation rates of adult females of Xenopsylla skrjabini and $X$. nuttali). Parazitologiya, 1971, 5, 137-139.

Gerasimova N. G., GAvrikova E. A. : (The length of life of the fleas Xenopsylla skrjabini Ioff and Xenopsylla nuttali Ioff). Parazitologiya, 1970, 4, 385-388.

Graves G. N., Bennett W. C., Wheeler J. R., Mrller E. B., Forcum D. L. : Sylvatic plague studies in southeast new Mexico. II - Relationships of the Desert Cottontail and its fleas. J. Med. Entomol., 1978, 14, 511-522.

Grenier P. : Contribution à l'étude des puces des mammifères sauvages de la forêt de Nefifik. Arch. Inst. Pasteur Maroc, 1957, 5, 206-217.

Holland G. P. : Primary and secondary sexual characteristics of some Ceratophyllinae, with notes on the mechanism of copulation (Siphonaptera). Trans. R. ent. Soc. Lond., 1955, 107, 233-248.

Hopkins G. H. E., Rothschild M. : An illustrated catalogue of the Rothschild collection of fleas (Siphonaptera) in the British Museum (Natural History). Vol. I : Tungidae and Pulicidae. British Museum, 1953, 36r p., I carte, $45 \mathrm{pl}$.

Humphries D. A. : The mating behaviour of the hen flea Ceratophyllus gallinae (Schrank) (Siphonaptera: Insecta). Anim. Behav., 1963, Is, 82-90.

KIR'yakova A. N., Koptzov L. A., Koptzova Z. G. : (The number of generation in fleas of the genus Xenopsylla within a year in northern Kizilkum). Parazitologiya, 1970, 4, 524-527.

KLeIN J. M. : Données faunistiques et écologiques sur les puces des mérions d'un foyer naturel de peste au Kurdistan iranien. Bull. Soc. Path. exot., 1963, 56, 1202-1230.

KLeIN J. M. : Données écologiques et biologiques sur Synopsyllus fonquerniei Wagner et Roubaud, I932 (Siphonaptera), puce du rat péridomestique, dans la région de Tananarive. Cah. O.R.S.T.O.M., Sér. Ent. Méd. Parasitol., 1966, 4, 3-29. 
Klein J. M., Hebrard G., Kim Suor : Étude écologique sur les puces des rats commensaux de l'homme et en particulier sur Xenopsylla cheopis (Roths.), 1903 (Siphonaptera), dans la région de Phnom-Penh (Cambodge). Cah. O.R.S.T.O.M., sér. Ent. Méd. Parasitol., 1973, II, 57-73.

Kunitskaya N. T. : (Sur les organes reproducteurs des puces femelles et la détermination de leur âge physiologique.) Méd. Parazitol., I960, 29, 688-701.

Kunitskaya N. T., Nunitsky V. N., Gauzshtein D. M., Savelova N. M. : (The physiological age of fleas and an analysis of the age composition of a natural population of $X$. gerbili Wagn.) Parazitologiya, 1977, II, 202-209.

LANe R.: The V.I.P. fleas from Spain. Ent. Month. Mag., 1966, 102, 211-216.

Launay H. : Approche d'une prophylaxie de la myxomatose : écologie des puces du lapin de garenne. Bull. mens. Off. Nation. Chasse, I981, No Sp. Scien. Techn.,. 213-24r.

MEAD-BRIGgS A. R. : The structure of the reproductive organs of the European Rabbit-flea, Spilopsyllus cuniculi (Dale) (Siphonaptera). Proc. R. ent. Soc. Lond. (A), 1962, 37, 79-88.

MeAd-Briggs A. R. : The reproductive biology of the rabbit flea Spilopsyllus cuniculi (Dale) and the dependence of this species upon the breeding of its host. J. Exp. Biol., I964, 4I, $37 \mathrm{I}-402$.

Mead-Briggs A. R. : The European Rabbit, the European Rabbit Flea and Myxomatosis. $A p p l$. Biol., I977, 2, 183-26I.

Mead-Briggs A. R., Vaughan J. A., Rennison B. D. : Seasonal variation in numbers of the rabbit flea on the wild rabbit. Parasitology, 1975, 70, 103-1 8 .

Prokopiev V. N. : (Méthodes de détermination de l'âge physiologique des femelles d'Oropsylla silantiewi Wag. et les variations saisonnières de la composition par âge dans de grandes populations). Isv. Irkutsk. Gosudar. Nautch. Issledov. Protiv. Ins. Sibiri i daln., Vostoka, I958, $17,9 \mathrm{I}-108$.

Rodriguez J. A. : Estudio de ectoparasitos de Leporidos en la provincia de Ciudad Real. Thèse Doct. Pharmacie, 1980, Madrid, 383 p.

Rothschild M., Ford B. : Breeding cycle of the flea Cediopsylla simplex is controlled by breeding cycle of host. Science, Wash., I972, $178,625-626$.

Rothschild M., Ford B., Hughes M. : Maturation of the male rabbit flea (Spilopsyllus cuniculi) and the oriental rat flea (Xenopsylla cheopis) : some effects of mammalian hormones on development and impregnation. Trans. Zool. Soc. Lond., I970, 32, 105-188.

Rousselle M. : A propos de quelques récoltes de Siphonaptères au Maroc. J. Med. Maroc, I968, s, I53-I6I.

Smit F. G. A. M. : A new species of Xenopsylla parasitic on rabbits in Morocco. Arch. Inst. Pasteur Maroc, 1957, S, 20I-205.

WILliams R. T.: Establishment and seasonal variation in abundance of the European rabbit flea, Spilopsyllus cuniculi (Dale), on wild rabbits in Australia. J. Ent. (A), 1973, 48, I1 7-127.

Yakunin B. M., Chernova N. A., Kunitskaya N. T. : (On the number of generations of the flea Xenopsylla skrjabini in Mangyslak (Aphaniptera). Parazitologiya, 1979, 13, 510-515.

Zolotova S. I., Afanas'eva O. V. : (On the biology of Xenopsylla gerbilli minax Jord., 1926). Parazitologiya, 1969, 3, 301-308.

Zolotova S. I., Maslennikova Z. P., Afanas'eva O. V. : (Number of generations of fleas of the genus Xenopsylla (Siphonaptera, Pulicidae) in the subzone of the northern deserts). Zool. Zh., 1978, s7, 1663-1667. 